\title{
Does the setting matter? Observing wheelchair transfers across different environmental conditions
}

\author{
Giulia Barbareschi*, Sharon Sonenblum $^{\mathrm{b}}$, Catherine Holloway ${ }^{\mathrm{a}}$, Stephen \\ Sprigle $^{\mathrm{b}}$
}

${ }^{a}$ University College London Interaction Centre, London, UK; ${ }^{b}$ Rehabilitation

Engineering and Applied Research, Georgia Institute of Technology, Atlanta (GA),USA

Giulia Barbareschi, University College London Interaction Centre, 66-72 Gower Street (2nd Floor), London, UK, WC1E 6EA. Tel +44 (0) 2031087192

Email giulia.barbareschi.14@ucl.ac.uk

\begin{abstract}
The setting in which wheelchair transfers are performed can affect difficulty and the risks associated with completion. This article presents results from an observational study involving 13 wheelchair users performing independent transfers across four settings. The aim is to understand how the environment affects how different types of independent transfers are performed. Descriptive analysis was performed alongside an objective assessment using the Transfer Assessment Instrument (TAI). The perceived difficulty reported after each transfer was also collected. Two participants exhibited radically different transferring techniques in different scenarios. Additionally, the transferring scenario was found to significantly affect the perceived difficulty of sitting transfers (toilet $2.17 \pm .88$; bed $1.47 \pm .65, \mathrm{p}=.001$; car $1.63 \pm .82, \mathrm{p}=.012$ ) and standing transfers (car $3.5 \pm .71$; bed $1 \pm 0, \mathrm{p}=.03$; toilet $1 \pm 0, \mathrm{p}=.03$ ), and the TAI score attributed to sitting pivot with use of a transfer board ( couch $4.3 \pm$ .88 ; bed $6.93 \pm 1.29, \mathrm{p}=.022$; car $7.13 \pm 1.32, \mathrm{p}=.018)$. Overall, environmental constraints, can lead to major technique changes and, more often, to different positioning of hands and feet which could impact the transfer's biomechanics.
\end{abstract}

Keywords: wheelchair users; wheelchair transfers; upper limb injury; activities of daily living

\section{Introduction}

Many Activities of Daily Living (ADLs) such as getting up from bed, taking a shower or having dinner with one's family take place in specific environments that often shape 
the way in which the person carries out the activity. The performance of general skills like wheelchair propulsion or transferring is usually aimed at the completion of various ADLs. The specific ADL will determine the physical context in which these skills are performed. In turn, the physical environment will affect the way in which the person propels (Hurd, Morrow, Kaufman, \& An, 2009) or transfers to and from the wheelchair (Toro, Koontz, \& Cooper, 2012). Studies looking at the characteristics of wheelchair propulsion, have shown how different types of environmental conditions, in the form of indoor and outdoor terrains, can greatly affect how a person propels the wheelchair and how much force is required when propelling (Hurd et al., 2009; Hurd, Morrow, Kaufman, \& An, 2008; Richter, Rodriguez, Woods, \& Axelson, 2007).

From a biomechanical point of view, wheelchair transfers are less constrained activities than wheelchair propulsion. In fact, during the push phase of wheelchair propulsion the person's hand is connected to the wheel's pushrim and the movement of the whole upper body will be dependent from the path followed by the hand. The recovery phase of propulsion is slightly less limited, but previous studies have shown that there is little variation in the movements observed across a multiple wheelchair users (Koontz et al., 2009). On the other hand, the path followed by the body during wheelchair transfers is subject to much larger variations with vastly different techniques observed across participants even when examining one type of transfer (Haubert et al., 2015). For this reason, it is reasonable to expect that environmental conditions will play an even bigger role in determining the movement strategy adopted by the individual and the potential risks associated with transfer performance. Furthermore, wheelchair transfers are also linked with the development of shoulder pain and injury among wheelchair users (Hogaboom et al., 2016). In particular, car transfers have been reported as one of the activities that is more often accompanied by higher intensity of shoulder pain (Alm et 
al., 2008).

Despite this, studies on wheelchair transfers have mainly been carried out within laboratory settings, often using sophisticated and expensive equipment and standardized protocols (Gagnon, Nadeau, Desjardins, \& Noreau, 2008; Kankipati, Boninger, Gagnon, Cooper, \& Koontz, 2015; Koontz, Gagnon, Brindle, \& Cooper, 2010; Koontz, Kankipati, Lin, Cooper, \& Boninger, 2011).

These studies focus on specific aspects of the independent sitting transferring technique and the effect of vertical and horizontal gaps between transfer surfaces. They conclude transfers using a head-hips technique (the person leans forward and pivots by turning the shoulder in the direction opposite to the direction of the transfer) compared with upright trunk technique (the person does not lean forward and keeps the shoulders in line with the buttocks when moving towards the target surface) (Kankipati et al., 2015; Koontz et al., 2011); and transfers between surfaces with minimal height difference and small horizontal gaps (Gagnon et al., 2008; Kankipati et al., 2015; Koontz et al., 2010) will help to reduce mechanical load and preserve upper limb function. Each of these studies comment on the importance of placing the leading hand close to the initial position of the body in order to ensure optimal technique. These laboratory-based studies offer great advantages in terms of accuracy of measurements, reproducibility and possibility of comparing results across different studies, however, these settings are often not representative of real life conditions (Crytzer, Cooper, Jerome, \& Koontz, 2015). This is important as the positioning of the leading hand is itself determined by the environment in which the transfer is taking place and can influence the strategy adopted by individuals. Only a few researchers have explored the characteristics of wheelchair transfers across different real life conditions (Haubert et al., 2015; Kataoka et al., 2012; Toro et al., 2012; Tsai et al., 2018). 
The study carried out by Haubert et al., (2015) describes the technique used by 29 participants with paraplegia when transferring and loading the wheelchair into and out of their vehicle. A great variability of leg and hand positioning was reported, resulting in changes to muscular activity and force generation. According to the authors, the body lift phase of the car transfer is the most physically demanding for the person and its duration is directly correlated with the level of SCI of the person and the height difference between the wheelchair and the vehicle seat. Haubert et al., (2015) also suggested that placing the leading hand on the vehicle seat rather than the steering wheel when transferring inside the vehicle might reduce the strain on the leading shoulder and decrease the likelihood of injury overtime. On the other hand, Kataoka et al., (2012) focused on the kinematic analysis of the movements used during car transfers by 4 individuals with tetraplegia using a transfer board. All participants demonstrated a rotatory head-hip relationship (head moves in the opposite direction of the buttocks) to facilitate the transfer. However, various degrees and combinations of trunk and neck flexion were used to successfully transfer (Kataoka et al., 2012). Finally, the study by Tsai, Boninger, Bass, \& Koontz, (2018) looked at the effect of two different toilet transfers configurations (front and side) on the transferring technique and associated upper limb load among 26 manual wheelchair users. Results showed that the front transfer setup allowed participants to perform better quality transfers that were associated with reduced upper limb loads. The only two studies with a broad scope of transfer conditions were a carried out by Toro et al., (2012) and Koontz et al., (2019). The first one investigated how factors such as horizontal and vertical gaps, obstacles and grab bars affected the ability of 120 individuals to perform wheelchair transfers (Toro et al., 2012). The results were aimed at the creation of more accessible environments and the authors do not provide any information on how different 
conditions affected technique or perceived difficulty of wheelchair transfers. On the other hand, Koontz et al., (2019) included measures of transfer quality using the Transfer Assessment Instrument and showed that the presence of grab bars and a backrest not only enables people to transfer between surfaces featuring higher gaps, but it also helps to improve technique. However, both these studies used artificial scenarios which might not be fully representative of real life conditions.

The aims of the authors in this study is to explore how wheelchair transfer technique changes across different transfer scenarios, moving beyond the traditional mat table used in many laboratory based transfer studies. The impact that different environmental conditions have on objectively measured transfer quality, subjective perception of difficulty, and the relationship between these two assessment tools are also explored. Findings from this study will help create a better understanding of how the movement strategies employed by wheelchair users are affected by the type of transfer they perform and how these changes can affect the difficulty of a transfer or the risks associated with its performance. Ultimately, the aim of this paper is to provide evidence for clinical recommendations that are more aware of the contextual nature of wheelchair transfers.

\section{Methods}

\section{Participants}

Participants were included in the study if they met the following inclusion criteria: (1) aged between 18 and 65 years, (2) used a wheelchair as primary means of mobility for at least 6 months and (3) had the ability to perform wheelchair transfers independently with or without a transfer board. Participants were excluded if they (1) were currently an inpatient in a medical facility or (2) reported any medical condition that could be 
exacerbated through the study protocol. In accordance with the protocol approved by the XXXX (removed for anonymization) IRB, participants were asked to sign informed consent prior to participation.

\section{Experimental procedure}

Four transfer scenarios were recreated for the study: car, toilet, bed and couch. All participants had to perform transfers in the bed scenario, where the height of the bed was set to match the height of the individual's wheelchair. This was done to reduce the level of difficulty as much as possible and ensure that all participants would be able to complete at least one scenario. Participants where then able to choose two out of the other three scenarios proposed, according to the types of transfers they performed more frequently. The order of the three scenarios was randomised for each participant. This approach was chosen as we found a significant variability in the transfers participants reported they performed in their daily lives. Indoor scenarios used in the study are shown in Figure 1. When a participant chose the car transfer scenario, the participant's own vehicle was used. Four participants drove a sport utility vehicle. However, only 2 (Participant 8 and 12) transferred directly onto the driver's seat while Participant 2 and 3 transferred onto a car lift (Figure 2). Four participants drove sedan vehicles, and two an adapted minivan fitted with a swivel seat and performed the transfers with the wheelchair inside the vehicle (Figure 2).

Figure 1. Indoor transfer scenarios

Figure 2. Car transfer scenarios

Each participant was asked to perform up to four transfers (two from the wheelchair onto the target surface and two from the target surface onto the wheelchair seat) for each scenario. Adequate resting periods were given to avoid fatigue. 


\section{Transfer strategy, height difference and perceived difficulty}

As participants used their own wheelchairs throughout the experiment, this resulted in different height gaps between the wheelchair seat, measured from the top of the pressure relief cushion, and the other transfer surface. Height differences between start and target seat were measured and assigned to one of three categories: less than $5 \mathrm{~cm}$, between 5 and $15 \mathrm{~cm}$ and greater than $15 \mathrm{~cm}$. Horizontal gaps were not measured as the scale used to evaluate transfer quality specifically accounts for it. Video recordings were collected using 2 USB Logitech C930e webcams (Logitech Europe S.A., CH) positioned at angles which allowed for all necessary views to be captured. Video recordings were used to analyse the transfer strategy adopted by participants, including hand and feet positioning, number of scoots and false starts. False starts were described as instances where the participant completely lifts the buttocks as for starting the transfer, but lands back on the start surface without completing the movement. At the end of each transfer, participants were asked to rate its difficulty using a modified Category-Ratio (CR) 10 Borg Scale (Borg, 1982).

\section{Evaluation of transfer quality}

The Transfer Assessment Instrument (TAI) is described as a tool that can be used to assess safety, strategies for conservation of the upper limbs and ability to direct caregivers during the performance of independent and assisted wheelchair transfers (McClure, Boninger, Ozawa, \& Koontz, 2011). Previous studies have shown that the version 3.0 of the TAI had acceptable to high levels of intra and interrater reliability (range, .74-.88) and that TAI scores are not biased by subjects demographic characteristics, showing good construct validity. Study participants were evaluated using only Part 1 of the TAI 3.0. This version of the TAI was preferred to more recent versions as it has been used by previous studies of similar nature (Hogaboom, Worobey, 
\& Boninger, 2016; Tsai et al., 2016; Tsai, Hogaboom, Boninger, \& Koontz, 2014). Part 1 of the TAI 3.0 is composed of 15 items with a dichotomous score concerning different aspects of transfer performance including wheelchair positioning and management, arm and hand position, and quality of body movement during transfer execution (Tsai, Rice, Hoelmer, Boninger, \& Koontz, 2013). Part 2 of the TAI was not used for the evaluation as it does not evaluate single transfers but a summary performance of 4 transfers carried out in the same conditions. Furthermore, the final score of the TAI, which includes both Part 1 and Part 2, has been shown to be highly correlated $(\mathrm{R}=.97)$ to the score of Part 1 (Tsai, Hogaboom, Boninger, \& Koontz, 2014).

Using the videos collected by the two cameras placed at different angles, transfers were assessed independently by two trained physiotherapists only using Part 1 of the TAI and disagreements over different scores was resolved through consensus meetings. Items 4 , 5 and 15 of the TAI were removed from the evaluation as they were not applicable to more than $80 \%$ of the transfers performed by participants.

\section{Statistical analysis}

Descriptive statistics were calculated for demographic data of participants. As the movement strategies adopted during transfers are highly dependent on the technique used by the individual (Barbareschi, Cheng, \& Holloway, 2017), transfers were assigned to three different categories according to the technique used by the individual: standing, sitting and sitting pivot with use of a transfer board and only compared within the same category.

The different environmental scenarios necessitated different height set ups, and the effect of both environmental scenarios and the height differences on the TAI scores were tested. All comparisons were based on transfer trials rather than participants' samples, meaning that if one participant performed two transfers with different 
technique, within the same scenario or height gap each individual transfer would have been assigned to a different group.

TAI scores were checked for normality using the Shapiro Wilk test. Due to the non-normality of the data four Kruskal-Walllis tests were first used to assess the impact of different transfer scenarios and height gaps on the TAI score and the self-reported perceived difficulty. Where significant differences were found a Dunn's test with Bonferroni correction was used for post-hoc pairwise comparison. No two-way analysis was attempted and each factor was examined separately. Spearman's correlation was used to investigate the presence of a linear relationship between TAI score and perceived difficulty. The level of significance for all tests was set at 0.05 . As the sample size for the study is small, both the effect size ( $\eta 2)$ and the statistical power were calculated and reported alongside each test performed. The statistical analysis was performed using SPSS 24 software (SPSS Inc., Chicago, IL, USA).

\section{Results}

\section{Participants}

Thirteen participants volunteered to take part in the study. A summary of demographic characteristics is provided in Table 1.

Table 1. Demographic characteristics of participants

\section{Transfer characteristics}

Data for 153 transfers were collected from 13 participants. Participant 1 only completed 1 of the 4 couch transfers, resulting in a total of 9 transfers. One hundred and twelve transfers were performed using a sitting technique, 26 with a standing technique and 15 sitting pivot with use of a transfer board. Sitting transfers where characterized by the fact that most of the weight of the person was borne by the upper limbs whereas the 
knees and hips remained flexed throughout the transfer. On the other hand, during standing transfers participants bore the majority of the weight on their lower limbs, progressively extended hips and knees after seat-off and flexed them again to sit on the target seat. Finally, during sitting pivot transfers with use of a transfer board most of the participant weight was placed on the board as the centre of mass moved between the initial and the target surface.

Only a few participants (Participant 1, 6, 7 and 11) stated that couch transfers were a regular occurrence when at home. Participant 3 and 10 stated that they avoid couch transfer at home as they find them difficult due to the low height of the couch, whereas other participants simply reported being more comfortable sitting on their wheelchair. Participant 1 and 11 stated that they used a commode at home rather than a standard toilet and did not feel comfortable performing unusual transfers as part of the study. Finally, Participant 6 and 7 did not own a private vehicles and reported car transfers as very rare occurrences, hence they chose not to perform car transfers as part of the study. An overview of the type of wheelchair used by participants for the study, the number of transfers they performed, the technique used and the scenarios in which the transfers were performed is illustrated in Table 2 .

Table 2. Technique, number and scenarios of wheelchair transfers performed by participants during the study

Transfer technique was consistent across scenarios for 11 participants. However, Participant 2 used a sitting technique for 10 transfers and a standing technique twice, when transferring from his wheelchair to the seat of his car lift. Participant 11 transferred 6 times with a transfer board, when transferring from the bed back onto his wheelchair and for all couch transfers, and 6 times without. Eighty-four of the transfers performed (52 bed, 12 toilet, $20 \mathrm{car}$ ) had a height gap of less than $5 \mathrm{~cm}$. For 61 of the 
transfers performed ( 32 toilet, 16 car, 13 couch), the height difference was between 5 and $15 \mathrm{~cm}$. The remaining 8 transfers (car) featured a height gap greater than $15 \mathrm{~cm}$.

\section{Effect of height gap, and scenario on transfer quality and difficulty}

Height gaps were only found to have a significant effect on TAI score $\left(\chi^{2}(1)=6.56, p=\right.$ $.01, \eta^{2}=.61$, power $\left.=.98\right)$ for sitting pivot transfers with use of a transfer board. The TAI score was significantly lower for transfers performed between surfaces featuring gaps of $5-15 \mathrm{~cm}(4.3 \pm .88, \mathrm{p}=.01)$ compared to level transfers $(7.01 \pm 1.23)$. No other significant difference of height gaps on TAI score was observed for transfers performed with a sitting $\left(p=.61, \eta^{2}=.01\right.$, power $\left.=.13\right)$ or standing technique $\left(p=.71, \eta^{2}=.03\right.$, power $=.14)$. Regardless of the transferring technique used, height gaps did not have any significant effect on the transfer reported difficulty (lowest $p=.1$ ). A summary of the impact of different height gaps on TAI score and reported transfer difficulty is shown in Table 3.

A significant difference in the reported difficulties among transfers performed in different scenarios was found for sitting $\left(\chi^{2}(2)=7.84, p=.001, \eta^{2}=.126\right.$, power $=.95$ ) and standing transfers $\left(\chi^{2}(3)=4.19, p=.017, \eta^{2}=.489\right.$, power $\left.=.78\right)$. Among participants who transferred with a sitting technique, toilet transfers were judged to be significantly more difficult $(2.17 \pm .88)$ than bed transfers $(1.47 \pm .65, \mathrm{p}=.001)$ and car transfers $(1.63 \pm .82, \mathrm{p}=.012)$. When transfers were performed with a standing technique, car transfers were found to be significantly more difficult $(3.5 \pm .71)$ than bed $(1 \pm 0, p=.03)$ and toilet transfers $(1 \pm 0, p=.03)$. Among transfers sitting pivot transfers completed with use of a transfer board, no significant differences were found in the reported difficulties across the different scenarios $\left(\mathrm{p}=.32, \eta^{2}=.184\right.$, power $=$ .22). However, the different scenarios had a significant effect on the TAI score attributed to sitting pivot transfers with use of a transfer board $\left(\chi^{2}(2)=6.629, p=.036\right.$, 
$\eta^{2}=.612$, power $\left.=.92\right)$. Post hoc analysis showed that couch transfers received significantly lower TAI scores $(4.3 \pm .88)$, compared to bed $(6.93 \pm 1.29, \mathrm{p}=.022)$ and car transfers $(7.13 \pm 1.32, \mathrm{p}=.018)$. A summary of the impact of different transfer scenarios on TAI score and reported transfer difficulty is shown in Table 4.

No significant correlation was found between TAI score and reported difficulty for sitting $\left(\mathrm{r}_{\mathrm{s}}(110)=.1, \mathrm{p}=.3\right)$, standing $\left(\mathrm{r}_{\mathrm{s}}(24)=.07, \mathrm{p}=.72\right)$ and sitting pivot transfers with use of a transfer board $\left(\mathrm{r}_{\mathrm{s}}(13)=-.21, \mathrm{p}=.45\right)$.

\section{Descriptive analysis}

Across transfers performed with a sitting technique, the overall strategy was similar for bed, toilet and car transfers where the vehicle was fitted with either a lift or a swivel seat. Across these scenarios, both leading and trailing hands were positioned on the transfer surface either flat or in a fist. In the toilet scenario where two handrails were available, participants utilized them for only $25 \%$ of transfers on the leading side and for $33.3 \%$ of transfers on the trailing side. Both feet were kept on the footrest for $10.8 \%$ of transfers, for $48.65 \%$ of transfers they were both placed on the ground and in the remaining cases one was placed on the ground while the other one was positioned on the footrest.

Movement strategies were different for transfers performed between the wheelchair and the driver seat of a sedan or sport utility vehicle. When exiting the vehicle, the leading hand was often placed onto the wheelchair cushion (70\%), whereas placements on the wheelchair frame (15\%) or the wheelchair's wheel (15\%) were less common). Similarly, when entering the vehicle, the leading hand was most commonly placed $(75 \%)$ on the driver's seat and less often on the steering wheel $(25 \%)$. When entering the vehicle, the trailing hand was often placed on the starting surface $(80 \%)$, and occasionally on the door of the vehicle $(20 \%)$. When exiting the vehicle, the trailing 
hand was also frequently placed on the starting surface $(65 \%)$. However, positioning the trailing hand on the steering wheel $22.5 \%$ was also reasonably common, whereas placement on top of the vehicle's door were more rare (12.5\%). When exiting the vehicle, participants almost always (80\%) positioned the leading foot on the ground ad left the trailing foot on the floor of the car. Occasionally both feet were placed on the floor of the car (17.5\%) and only in one occasion (2.5\%) both feet were placed on the ground. When transferring from the wheelchair to the driver's seat, participants were equally likely to place the leading foot inside the car and the trailing foot on the ground or to place both feet on the ground (37.5\%). In the remaining cases participants placed booth feet inside the car before entering the vehicle (25\%). Participants performed a scoot to move towards the front of the seat for $60.7 \%$ of the transfers. False starts were observed for $3.6 \%$ of transfers.

The movement strategies adopted by participants performing standing transfers did not appear to vary across different scenarios. Both feet were stably placed on the ground and, when available, on both leading (62.8\%) and trailing side (71.4\%) participants preferred to place their hands on structures that could be easily grabbed to increase stability and facilitate lift and descent as shown in Figure 4. Additionally, hand positions often changed within the same transfer following the body movement to help increase support. Scoots were observed for $23.1 \%$ of the transfers and false starts for $11.1 \%$ of the transfers. Both scoots and false starts were more common when the starting surface was soft and offered low support but increased friction (i.e.: bed, couch).

Among sitting pivot transfers with use of a transfer board, movement strategies depended on the direction of the transfer (to/from the wheelchair) rather than the scenario. When transferring out of the wheelchair, participants maintained their feet on 
the footrest, whereas one foot was positioned on the footrest and the other on the ground when the participant was transferring back onto the wheelchair. The trailing hand was usually placed flat on the starting surface across all scenarios and progressively slid across the transfer board as the subject moved towards the target surface. In contrast, the position of the leading hand was greatly affected by the transferring scenario. During bed and couch transfers the leading hand was always placed flat on the transfer board, during car transfers participants used the leading hand to grab the steering wheel or the overhead handle to pull themselves towards the target surface. Scoots were observed for $26.6 \%$ of transfers, and false starts for $33.3 \%$ of transfers. All occurrences of scoots and false starts were detected during couch and bed transfers.

\section{Discussion}

This is the first study to analyse quality and perceived difficulty of wheelchair transfers across various scenarios for participants using different transferring techniques. Transferring techniques observed across the participants were: independent seated transfer, sitting pivot transfer with use of a transfer board and standing transfer, eleven participants exhibited only one technique while two participants demonstrated two different techniques across the 12 transfers performed. This is particularly interesting as these changes in technique were highly dependent on the settings in which the transfers were performed. Participant 2 was only able to perform standing transfers when moving from his wheelchair to the vehicle's seat as he grabbed the top of the vehicle's door and used a custom mounted grab bar to gather the support necessary to reach a standing position. Similarly, Participant 11 was able to perform transfers using an independent sitting technique only for car transfers and transfers from the wheelchair to the bed. Both the presence of a height gap between the his wheelchair and the couch and soft starting surfaces that offered less support but had higher friction, such as bed's 
mattresses or couch's cushions, represented environmental barriers that caused him to need a transfer board to perform the transfer. Interestingly, participants' transferring technique was mostly similar across multiple transfers performed in the same scenario. However, variations in hands and feet positioning were often observed as participants would not always place the wheelchair in the exact same position and they adjusted their movement strategies.

Previous research (Barbareschi \& Holloway, 2018) highlighted how the transferring technique used individuals was primarily dictated by the person's medical condition, and that the use of assistive technologies (AT)s was linked to greater body weight. Our findings evolve the number of factors which can effect a person's transferring technique. Specifically, we show that environmental conditions can represent either a barrier or a facilitator of transfer performance with important effects on the individual's transferring technique and the use of ATs.

The presence of height gaps between transferring surfaces increases the upper limb mechanical load associated with the performance of wheelchair transfers (Gagnon et al., 2009; Toro et al., 2012). Nevertheless, in the current study, the presence of a greater height gap was only found to have a negative effect on the TAI score attributed to sitting pivot transfers performed with a transfer board. Height gaps are likely to present a greater challenge when participants have limited transferring ability and normally people would then use a transfer board. Participants in the current study were all expert wheelchair users with considerable transferring skills which could potentially explain why the reported difficulty and TAI scores attributed to transfers performed with sitting and standing techniques were not affected by the presence of height gaps.

Transferring scenario was found to significantly affect either the perceived difficulty or the TAI score, depending on the transferring technique. Participants 
performing standing transfers rated car transfers as more difficult. By contrast, when participants used a sitting technique bathroom transfers were found to be more challenging. This was particularly interesting as the accessible toilet used for this study was fitted with grab bars that are supposed to facilitate the performance of wheelchair transfers. However, as noted by Toro et al., (2012) and Tsai et al., (2018), grab bars commonly fitted in accessible toilets are often too high to be useful to people performing sitting transfers and were scarcely used by participants.

Interestingly, participants' familiarity with the transfer scenario did not also seem to play a role in neither the reported difficulty nor the TAI score attributed to participants' transfers. Indeed, transfers to and from their own vehicle, the only set up in the study that participants were familiar with, was not necessarily reported as the easiest nor the one featuring the highest TAI scores. In fact participants, using standing technique found car transfers the hardest to perform and participants using a sitting technique received slightly lower TAI score for car transfers compared to toilet and bed transfers.

Overall, indoor scenarios resulted in similar characteristics between participants, whereas car transfers presented strategies that where considerably different, due to the uniqueness of the set up. This potentially reinforces the findings from previous research that highlights the importance of further research into car transfers (Haubert et al., 2015; Kataoka et al., 2012; Schaupp et al., 2016). However, it is worth noticing that this was influenced by the study design as indoor scenarios where standardised across participants, whereas the vehicles used for the car transfers were different for each individual.

Interestingly, we were unable to find any significant relationship between the TAI score attributed by clinicians and the perceived difficulty reported by participants. This was 
surprising as previous studies by Newton, Kirby, MacPhee, Dupuis, \& MacLeod, (2002) and Rushton, Kirby, \& Miller, (2012), found a strong correlation between the subjective estimation of effort and the objective evaluation of individual's capacity across several wheelchair skills. However, Rushton et al., (2012) found that selfassessment of safety did not correspond to objective evaluation by trained professionals. As transfers and other wheelchair skills become an integral part of the daily routine, individuals could become used to performing these tasks with strategies that are nonefficient or potentially dangerous. Overall, our results show that to gather accurate insights from studies looking at the performance of wheelchair transfers, objective tools should be used to assess transfers' quality.

\section{Limitations}

Although this study illustrates novel insights concerning the performance of wheelchair transfers across different scenarios, it has limitations that need to be considered when interpreting the results. The small number of participants, although not uncommon among studies focussing on wheelchair users, mean caution should be taken in generalization of the results. Furthermore, the majority of participants had a spinal cord injury (SCI) which creates additional challenges for generalization. Additionally, due to the design of the study featuring the performance of repeated transfers, recruited wheelchair users potentially had above average transferring ability. Although the scenarios featured in the study are found in the real world, the indoor scenarios might be different from the one found in participants' houses, whereas for car transfers participants were allowed to use their own vehicles. Although all participants only performed transfers that they felt confident about and that they reported doing routinely (i.e. people who performed toilet transfers said that they commonly use public toilets with similar layouts), the reduced familiarity they had with indoor scenarios compared 
to their own vehicles, could have affected the reported difficulty. Some participants also had adapted vehicles featuring transfer lifts and swivel seats which could have made the transfer easier to perform. However, the perception of difficulty is very relative and participants who were used to their vehicles without adaptations might find the car transfers they routinely perform easier.

The physiotherapists evaluating participants' transfers in the current study also expressed concerns about the validity of the TAI with respect to the different scenarios as items related to upper limb positioning were judged to be less applicable for transfers performed to and from the vehicle due to specific environmental constraints.

Furthermore the original studies used to validate the TAI (Tsai et al., 2013) were carried out using a height adjustable mat, whereas the transfer scenarios used for the current study used a combination of different surfaces.

Finally, it is worth noting that the choice of grouping transfers by technique rather than participant led to consider each individual transfer as an independent instance, despite the fact that each participant performed more than one transfer in each scenario or at a particular height gap. Hence, the Kruskal-Wallis test used to measure the effect of height gap and scenarios on TAI score and reported difficulty assumption of independence could have been violated in some instances, especially for transfers performed with a transfer board that were carried out only by two participants, which suggests caution in the interpretation of results.

\section{Conclusions}

Environmental constraints associated with various wheelchair transfers scenarios can lead to considerable changes in the movement strategy and the quality of the transfer, potentially increasing or reducing the risks of falls and upper limb injuries. This is particularly true for car transfers, as the configuration of different vehicles can create 
unique set ups that will determine how individuals perform transfers. The lack of a significant relationship between the perceived difficulty and the objective quality of the transfer could be due to the fact that "incorrect movement strategies" become part of the individual technique among participants living in the community.

\section{References}

Alm, M., Saraste, H., \& Norrbrink, C. (2008). Shoulder Pain in Persons with Thoracic Spinal Cord Injury: Prevalence and Characteristics. Journal of Rehabilitation Medicine, 40(4), 277-283. https://doi.org/10.2340/16501977-0173

Barbareschi, G., Cheng, T.-J., \& Holloway, C. (2017). The effect of technique and transfer board use on the performance of wheelchair transfers. Healthcare Technology Letters. https://doi.org/10.1049/htl.2017.0075

Barbareschi, G., \& Holloway, C. (2018). An investigation of factors affecting the performance of wheelchair transfers. Disability and Rehabilitation: Assistive Technology, O(0), 1-10. https://doi.org/10.1080/17483107.2018.1463402

Borg, G. (1982). A category scale with ratio properties for intermodal and interindividual comparisons. Psychophysical Judgment and the Process of Perception, 25-34.

Crytzer, T. M., Cooper, R., Jerome, G., \& Koontz, A. (2015). Identifying research needs for wheelchair transfers in the built environment. Disability and Rehabilitation: Assistive Technology, 0(0), 1-7. https://doi.org/10.3109/17483107.2015.1042079

Gagnon, D., Koontz, A., Mulroy, S., Nawoczenski, D., Butler-Forslund, E., Granstrom, A., Nadeau, S., \& Boninger, M. (2009). Biomechanics of Sitting Pivot Transfers Among Individuals with a Spinal Cord Injury: A Review of the Current 
Knowledge. Topics in Spinal Cord Injury Rehabilitation, 15(2), 33-58.

https://doi.org/10.1310/sci1502-33

Gagnon, D., Nadeau, S., Desjardins, P., \& Noreau, L. (2008). Biomechanical assessment of sitting pivot transfer tasks using a newly developed instrumented transfer system among long-term wheelchair users. Journal of Biomechanics, 41(5), 1104-1110. https://doi.org/10.1016/j.jbiomech.2007.11.021

Haubert, L. L., Mulroy, S. J., Hatchett, P. E., Eberly, V. J., Maneekobkunwong, S., Gronley, J. K., \& Requejo, P. S. (2015). Car Transfer and Wheelchair Loading Techniques in Independent Drivers with Paraplegia. Frontiers in Bioengineering and Biotechnology, 3. https://doi.org/10.3389/fbioe.2015.00139

Hogaboom, N. S., Worobey, L. A., \& Boninger, M. L. (2016). Transfer Technique Is Associated With Shoulder Pain and Pathology in People With Spinal Cord Injury: A Cross-Sectional Investigation. Archives of Physical Medicine and Rehabilitation, 97(10), 1770-1776. https://doi.org/10.1016/j.apmr.2016.03.026

Hurd, W. J., Morrow, M. M. B., Kaufman, K. R., \& An, K.-N. (2009). Wheelchair propulsion demands during outdoor community ambulation. Journal of Electromyography and Kinesiology, 19(5), 942-947. https://doi.org/10.1016/j.jelekin.2008.05.001

Hurd, W. J., Morrow, M. M., Kaufman, K. R., \& An, K.-N. (2008). Biomechanic Evaluation of Upper-Extremity Symmetry During Manual Wheelchair Propulsion Over Varied Terrain. Archives of Physical Medicine and Rehabilitation, 89(10), 1996-2002. https://doi.org/10.1016/j.apmr.2008.03.020

Kankipati, P., Boninger, M. L., Gagnon, D., Cooper, R. A., \& Koontz, A. M. (2015). Upper limb joint kinetics of three sitting pivot wheelchair transfer techniques in 
individuals with spinal cord injury. The Journal of Spinal Cord Medicine, 38(4), 485-497. https://doi.org/10.1179/2045772314Y.0000000258

Kataoka, M., Yasuda, T., Kataoka, T., Ueda, E., Yonetsu, R., \& Okuda, K. (2012). Movement strategies during car transfers in individuals with tetraplegia: A preliminary study. Spinal Cord, 50(6), 440-445. https://doi.org/10.1038/sc.2011.170

Koontz, A. M., Bass, S., Kulich, H., \& Cooper, R. A. (2019). Effects of grab bars and backrests on independent wheelchair transfer performance and technique. Physiotherapy Research International, 24(1), e1758.

Koontz, A. M., Gagnon, D., Brindle, E., \& Cooper, R. A. (2010). Positioning the wheelchair closer to the target surface reduces shoulder muscular demand for sitting pivot transfers. Rehabilitation: Mobility, Exercise, and Sports: 4th International State-of-the-Art Congress, 26, 13.

Koontz, A. M., Kankipati, P., Lin, Y.-S., Cooper, R. A., \& Boninger, M. L. (2011). Upper limb kinetic analysis of three sitting pivot wheelchair transfer techniques. Clinical Biomechanics, 26(9), 923-929. https://doi.org/10.1016/j.clinbiomech.2011.05.005

Koontz, A. M., Roche, B. M., Collinger, J. L., Cooper, R. A., \& Boninger, M. L. (2009). Manual Wheelchair Propulsion Patterns on Natural Surfaces During Start-Up Propulsion. Archives of Physical Medicine and Rehabilitation, 90(11), 1916-1923. https://doi.org/10.1016/j.apmr.2009.05.022

McClure, L. A., Boninger, M. L., Ozawa, H., \& Koontz, A. (2011). Reliability and Validity Analysis of the Transfer Assessment Instrument. Archives of Physical Medicine and Rehabilitation, 92(3), 499-508. https://doi.org/10.1016/j.apmr.2010.07.231 
Newton, A. M., Kirby, R. L., MacPhee, A. H., Dupuis, D. J., \& MacLeod, D. A. (2002). Evaluation of manual wheelchair skills: Is objective testing necessary or would subjective estimates suffice? Archives of Physical Medicine and Rehabilitation, 83(9), 1295-1299. https://doi.org/10.1053/apmr.2002.33071

Richter, W. M., Rodriguez, R., Woods, K. R., \& Axelson, P. W. (2007). Stroke Pattern and Handrim Biomechanics for Level and Uphill Wheelchair Propulsion at SelfSelected Speeds. Archives of Physical Medicine and Rehabilitation, 88(1), 8187. https://doi.org/10.1016/j.apmr.2006.09.017

Rushton, P. W., Kirby, R. L., \& Miller, W. C. (2012). Manual Wheelchair Skills: Objective Testing Versus Subjective Questionnaire. Archives of Physical Medicine and Rehabilitation, 93(12), 2313-2318.

https://doi.org/10.1016/j.apmr.2012.06.007

Schaupp, G., Seeanner, J., Jenkins, C., Manganelli, J., Hennessy, S., Truesdail, C., Swift, L., Venhovens, P., \& Brooks, J. (2016, April 5). Wheelchair Users' Ingress/Egress Strategies While Transferring Into and Out of a Vehicle. https://doi.org/10.4271/2016-01-1433

Toro, M. L., Koontz, A. M., \& Cooper, R. A. (2012). The Impact of Transfer Setup on the Performance of Independent Wheelchair Transfers. Human Factors: The Journal of the Human Factors and Ergonomics Society, 0018720812460549. https://doi.org/10.1177/0018720812460549

Tsai, C.-Y., Boninger, M. L., Bass, S. R., \& Koontz, A. M. (2018). Upper-limb biomechanical analysis of wheelchair transfer techniques in two toilet configurations. Clinical Biomechanics, 55, 79-85. https://doi.org/10.1016/j.clinbiomech.2018.04.008 
Tsai, C.-Y., Boninger, M. L., Hastings, J., Cooper, R. A., Rice, L., \& Koontz, A. M. (2016). Immediate Biomechanical Implications of Transfer Component Skills Training on Independent Wheelchair Transfers. Archives of Physical Medicine and Rehabilitation, 97(10), 1785-1792.

https://doi.org/10.1016/j.apmr.2016.03.009

Tsai, C.-Y., Hogaboom, N. S., Boninger, M. L., \& Koontz, A. M. (2014). The

Relationship between Independent Transfer Skills and Upper Limb Kinetics in Wheelchair Users. BioMed Research International, 2014, e984526.

https://doi.org/10.1155/2014/984526

Tsai, C.-Y., Rice, L. A., Hoelmer, C., Boninger, M. L., \& Koontz, A. M. (2013). Basic Psychometric Properties of the Transfer Assessment Instrument (Version 3.0). Archives of Physical Medicine and Rehabilitation, 94(12), 2456-2464.

https://doi.org/10.1016/j.apmr.2013.05.001 